\title{
Anesthesia Patient Monitoring and Control in Wireless-Based Systems
}

\author{
Zhibin Tan \\ Department of Electrical and \\ Computer Engineering \\ Wayne State University \\ Detroit, Michigan 48202 \\ Email: au6063@wayne.edu
}

\author{
Le Yi Wang \\ Department of Electrical and \\ Computer Engineering \\ Wayne State University \\ Detroit, Michigan 48202 \\ Email: lywang@wayne.edu
}

\author{
Hong Wang \\ Department of Anesthesiology \\ Wayne State University \\ Detroit, Michigan 48202 \\ Email: howang@med.wayne.edu
}

\begin{abstract}
This paper investigates impact of noise and signal averaging on patient control in anesthesia applications in wireless connected systems. Such systems involve communication channels which introduce noises due to quantization, channel noises, and have limited communication bandwidth resources. Usually signal averaging can be used effectively in reducing noise effects when remote monitoring and diagnosis are involved. However, when feedback is intended, we show that signal averaging will lose its utility substantially. To explain this phenomenon, we analyze stability margins under signal averaging and derive some optimal strategies for selecting windows size. A typical case of anesthesia depth control problems is used in this development.
\end{abstract}

\section{INTRODUCTION}

This paper investigates impact of communication channels and signal averaging on patient control in anesthesia applications in networked control system settings such as wireless connected systems, sensor networks, local area networks, or tele-medicine over a wide area network. When signals of a patient's vital signs must be transmitted through a communication channel, they will be subject to quantization and transmission errors, hence reducing the accuracy of signals for monitoring, diagnosis, and control.

To reduce the noise effect and improve the accuracy of estimated symbols, an averaging window is usually applied [1], [2]. It can be used effectively when remote monitoring and diagnosis are involved. However, signal averaging introduces dynamic delays. When feedback control is intended, such delays will have detrimental effects on closed-loop systems, even destabilizing the system. Consequently, signal averaging encounters a fundamental performance limitation in feedback systems. To explain this phenomenon, we analyze stability margins under signal averaging and derive some optimal strategies for selecting window sizes. A typical case of anesthesia depth control problems is used in this development.

The paper is organized as follows. Section II discusses patient modeling and feedback control in anesthesia applications. Signal averaging and its effectiveness on open-loop and closed-loop applications are demonstrated in Section III. The idea of using fast sampling is also discussed in this section. Theoretical foundation of our performance analysis is presented in Section IV. Our findings are applied to anesthesia U.S. Government work not protected by U.S. copyright control problems in Section V. Finally, Section VI summarizes some issues that are related but not resolved in this paper.

\section{Patient Models And Feedback Control}

\section{A. Patient Models}

For the purpose of predicting anesthesia patient responses to input drugs to perform control, predictive diagnosis, etc., much effort has been done to establish a reliable patient model that relates the drug or procedure inputs to the outcomes [3], [4], [6], [14]. Due to significant deviations in physical conditions, ages, metabolism, pre-existing medical conditions, and surgical procedures, patient dynamics demonstrate nonlinearity and large variations in their responses to drug infusion. So, it is necessary to establish a model in real-time and in individual patients. A basic information-oriented model structure for patient responses to drug infusion was introduced in [10]-[12].

Clinical data were collected to establish the patient model. One of these data sets is used in this paper. The anesthesia process lasted about 76 minutes, starting from the initial drug administration and continuing until last dose of administration. The patient's anesthesia depth is measured by a BIS (BiSpectrum) monitor [5], [9]. The monitor provides continuously an index in the range of $[0,100]$ such that the lower the index value, the deeper the anesthesia state. Propofol, a common anesthesia drug, was used in both titration and bolus which is adminstrated by an infusion pump. Fentanyl, a drug which is usually used to speed up the procedure of loss of consciousness for a patient, was injected in small bolus amount three times, two at the initial surgical preparation and one near incision. The patient was given bolus injection twice to induce anesthesia, first at $t=3$ minute with $20 \mathrm{mg}$ and then at $t=5$ minute with $20 \mathrm{mg}$. They are shown in Figure 1 as 10000 $\mu \mathrm{g} / \mathrm{sec}$ for two seconds, to be consistent with the titration units. The surgical procedures were manually recorded. Three major types of stimulation were identified: (1) During the initial drug administration (the first 6 minutes), due to set-up stimulation and patient nervousness. (2) Incision at $t=45$ minute for about 5 minutes duration. (3) Closing near the end of the surgery at $t=60$ minute. Analysis shows that the impact of Fentanyl on the BIS values is minimal. As a result, it is treated as a disturbance and not explicitly modeled in this example. 
The drug infusion was controlled manually by an experienced anesthesiologist. The trajectories of titration (in $\mu g / \mathrm{sec}$ ) and bolus injection (converted to $\mu \mathrm{g} / \mathrm{sec}$ ) during the entire surgical procedure were recorded, which are shown together with the corresponding BIS values in Figure 1(a).

The data from the first 30 minutes are used to determine model parameters and function forms. For estimating the parameters in the patient block, the data in the interval where the bolus and stimulation impact is minimal (between $t=10$ to $t=30$ minutes) are used. The patient model parameters was identified through Least-Squares estimation method [7].

Under a sampling interval $T=1$ second, which is the standard data transfer interval for the BIS monitor, the combined linear dynamics was estimated. The patient model with propofal infusion rate as the input and BIS measurement as the output was identified as

$$
P(z)=\frac{A(z)}{B(z)}
$$

,with sampling interval $T=1$ second. Where,

$$
A(z)=0.01872 z^{2}-0.08813 z+0.09016,
$$

$B(z)=z^{5}-1.159 z^{4}+0.7501 z^{3}-0.5989 z^{2}+0.2984 z-0.2678$

The actual BIS response is then compared to the model response over the entire surgical procedure. Comparison results are demonstrated in Figure 1(b). The model output represents the patient response very well. In particular, the model captures the key trends and magnitudes of the BIS variations in the surgical procedure. This indicates that the model structure contains sufficient freedom in representing the main features of the patient response.

\section{B. Feedback Control}

Usually to eliminate steady-state error in tracking control, an integrator is inserted into the system

$$
C(z)=\frac{1}{z-1} .
$$

A stabilizing feedback controller is designed for the patient model (1) by using a full-order observer and pole placement design

$$
F(z)=\frac{N(z)}{D(z)}
$$

, where

$$
\begin{aligned}
N(z)= & 1.234 z^{5}+0.6298 z^{4}-3.644 z^{3}+3.67 z^{2} \\
& -1.981 z+0.2479
\end{aligned}
$$

$$
\begin{aligned}
D(z)= & z^{6}-2.341 z^{5}+2.284 z^{4}-0.7252 z^{3} \\
& -0.4057 z^{2}+0.5714 z-0.08343
\end{aligned}
$$

These result in a combined system

$$
G(z)=F(z) C(z) P(z) .
$$
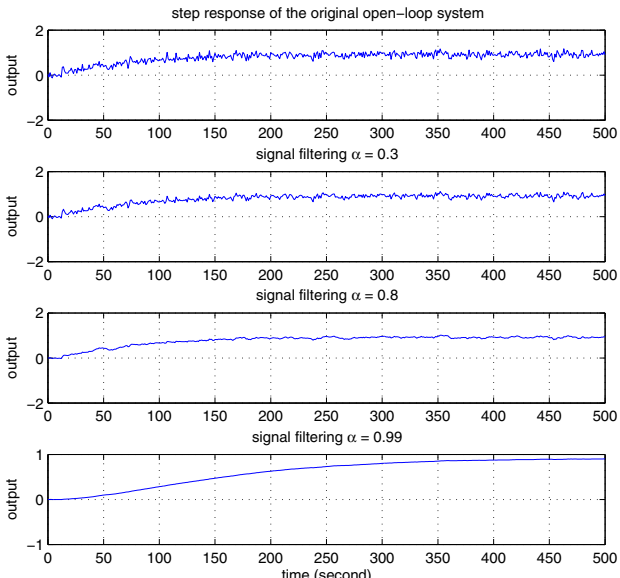

Fig. 3. Effects of signal averaging on open-loop systems

\section{Signal Averaging And Control Performance}

\section{A. Signal averaging}

An exponential averaging window with decaying weighting of rate $0<\alpha<1$ is

$$
h_{k}=(1-\alpha) \sum_{i=-\infty}^{k} \alpha^{k-i} x_{i}
$$

whose transfer function is

$$
F_{\alpha}(z)=\frac{(1-\alpha) z}{z-\alpha} .
$$

\section{B. Open-Loop Systems}

When signal averaging is applied to reduce noise effects, the resulting open-loop system can be represented by the block diagram in Figure 2(a).

Figure 3 illustrates impact of filtering on open loop systems. It is apparent that the longer the averaging window, the less the noise effect on the signal. On the other hand, filtering introduces a dynamic delay which has important implication on closed-loop applications.

\section{Closed-Loop Systems}

The closed-loop system with an averaging filter is shown in Figure 2(b).

The close-loop system equations are:

$$
y_{k}=G e_{k}, \quad e_{k}=r_{k}-F_{\alpha}\left(y_{k}+d_{k}\right)
$$

then,

$$
y_{k}=H_{r} r_{k}+H_{\alpha} d_{k}
$$

where,

$$
H_{r}=\frac{G}{1+F_{\alpha} G}, \quad H_{\alpha}=\frac{-F_{\alpha} G}{1+F_{\alpha} G}
$$

Figure 4 illustrates impact of filtering on closed loop systems. When filtering window is long, the filter can destabilize the closed-loop system. 

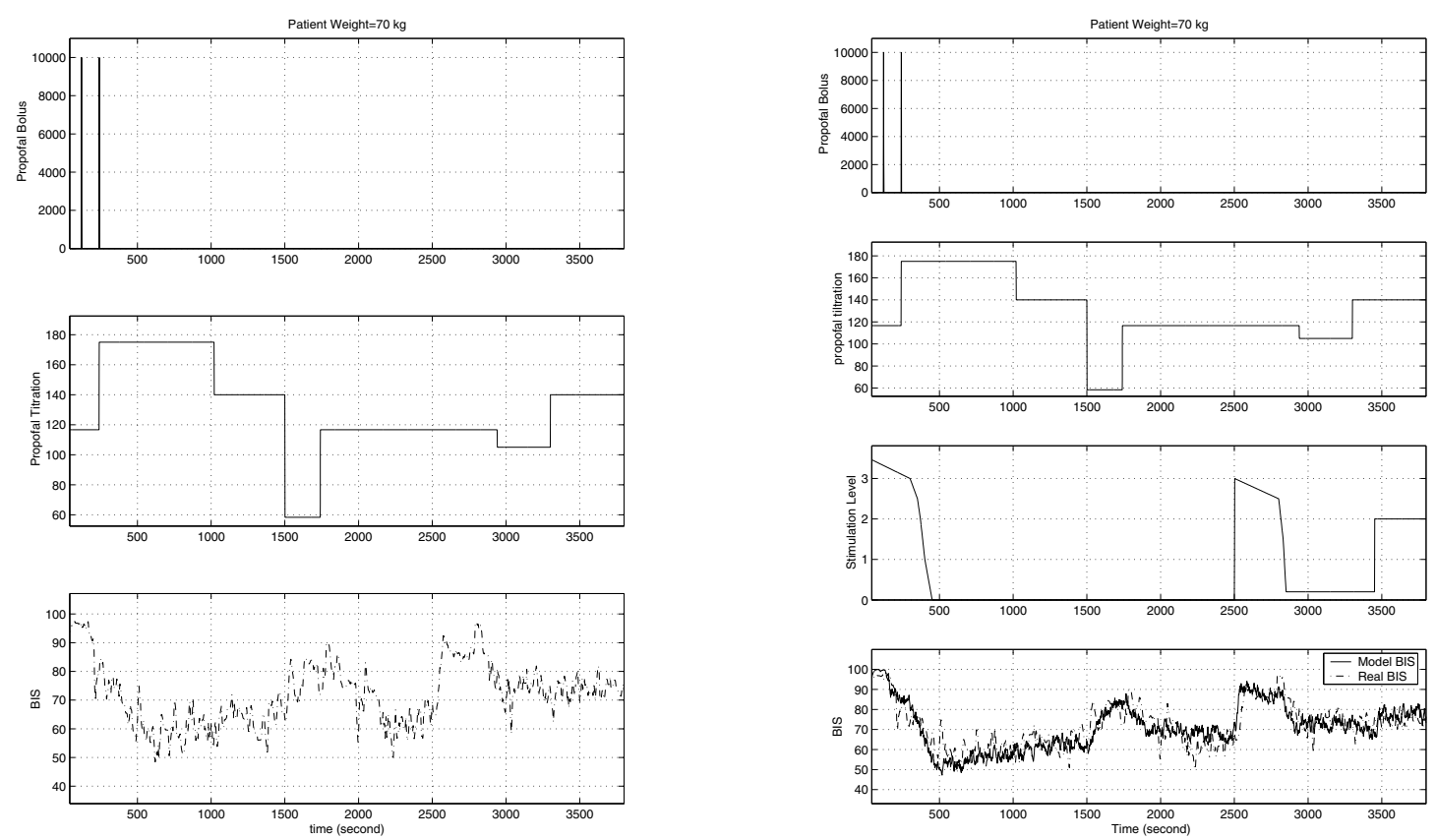

(a) Actual patient responses
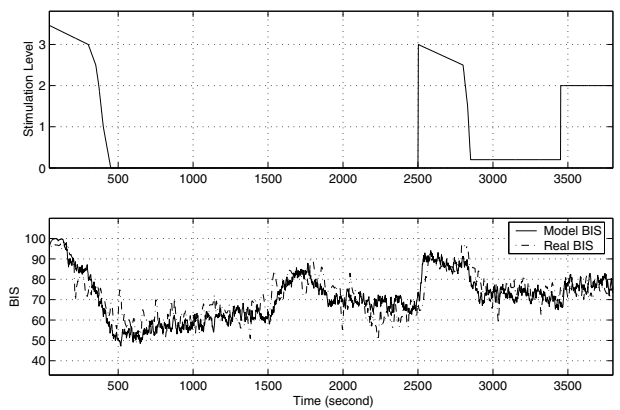

(b) Patient model responses

Fig. 1. Actual patient responses and patient model responses

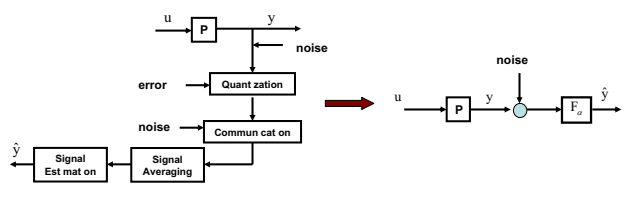

(a) Open-loop system module

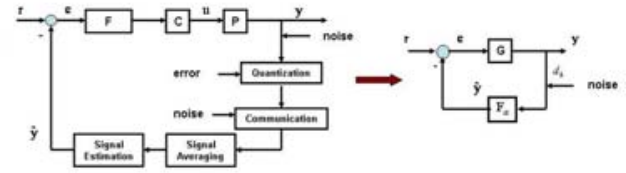

(b) Close-loop system module

Fig. 2. Signal filtering in open-loop and close-loop systems
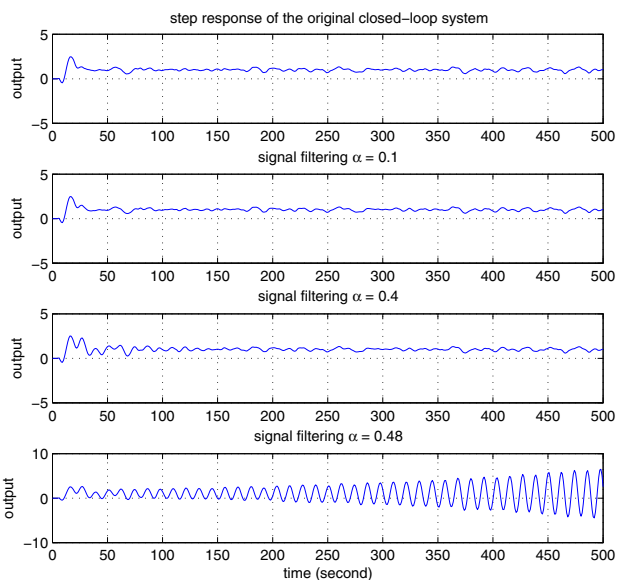

Fig. 4. Effects of signal averaging on closed-loop systems

\section{Re-Sampling}

The patient model (1) can be well approximated by a continuous-time system, $P(s)=e^{-5 s} \frac{0.93}{73 s+1}$, which consists of a pure time delay and a first-order dynamics, sampled with

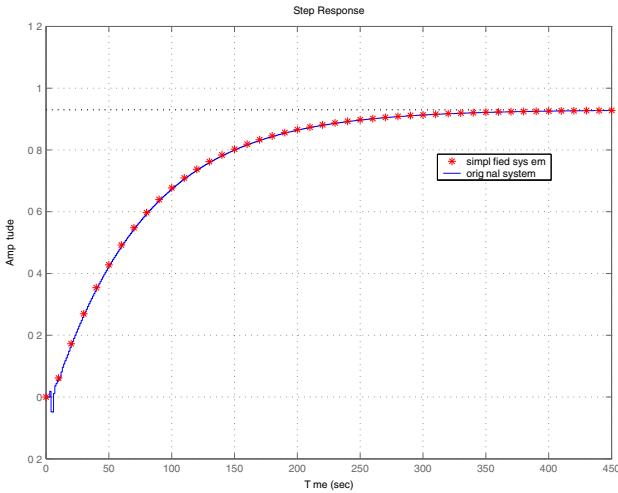

Fig. 5. Step responses of the original system and the simplified system

sampling interval $T=1$ second. The step responses of the original system (1) and the simplified system $P(s)$ are shown in Figure 5.

This approximation allows us to use smaller sampling intervals to re-sample the output of the system. The benefits of re-sampling will become clear after some theoretical analysis 
in the next section.

\section{Analysis of Stability and Performance}

\section{A. Feedback Robustness against Signal Averaging}

Definition 1: The stability margin against exponential averaging, abbreviated as $\alpha$-margin and denoted by $\alpha_{\max }(G)$, is the largest $0 \leq \alpha \leq 1$ such that for all $0 \leq \alpha<\alpha_{\max }(G)$, the close-loop system (6) is stable and the system is unstable if $\alpha>\alpha_{\max }(G)$. If the close-loop system is stable for all $\alpha$, we denote $\alpha_{\max }(G)=1$.

\section{B. Discrete and Continuous Time Averaging}

For an discrete time exponential averaging window:

$$
h_{k}=(1-\alpha) \sum_{i=-\infty}^{k} \alpha^{k-i} x_{i}=\theta+(1-\alpha) \sum_{i=-\infty}^{k} \alpha^{k-i} d_{i}=\theta+\varepsilon_{k} \text {. }
$$

If $d_{i}$ is i.i.d. with $E d_{i}=0$ and $E d_{i}^{2}=\sigma^{2}$, then

$$
E \varepsilon_{k}^{2}=\frac{1-\alpha}{1+\alpha} \sigma^{2}
$$

Consequently, using $h_{k}$ as an estimate of $\theta$ can reduce errors by $\frac{1-\alpha}{1+\alpha}$.

An continuous time exponential filter:

$$
F(s)=\frac{1}{\lambda s+1}
$$

whose impulse response is

$$
f(t)=\frac{1}{\lambda} e^{-t / \lambda}, t \geq 0
$$

Now,

$$
y(t)=\int_{-\infty}^{t} f(t-\tau) x(\tau) d \tau=\frac{1}{\lambda} \int_{-\infty}^{t} e^{-(t-\tau) / \lambda} x(\tau) d \tau
$$

For small $T, y(t)$ is approximated by

$$
\begin{aligned}
y_{k} & =y(k T)=\frac{1}{\lambda} \int_{-\infty}^{t} e^{-(t-\tau) / \lambda} x(\tau) d \tau \\
& \approx \frac{T}{\lambda} \sum_{i=-\infty}^{k}\left(e^{-T / \lambda}\right)^{k-i} x_{i} \\
& =\frac{T}{\lambda(1-\alpha)}(1-\alpha) \sum_{i=-\infty}^{k} \alpha^{k-i} x_{i} \\
& \approx(1-\alpha) \sum_{i=-\infty}^{k} \alpha^{k-i} x_{i}
\end{aligned}
$$

Where, $\alpha=e^{-T / \lambda}$.

\section{Stability Margin against Exponential Averaging}

Theorem 1: If the exponential stability margin in the continuous-time domain is $\lambda_{\max }$, then

$$
\lim _{T \rightarrow 0} \frac{T}{-\ln \alpha_{\max }}=\lambda_{\max } .
$$

Proof: This follows from the relationship

$$
\alpha=e^{-T / \lambda} \text {. }
$$

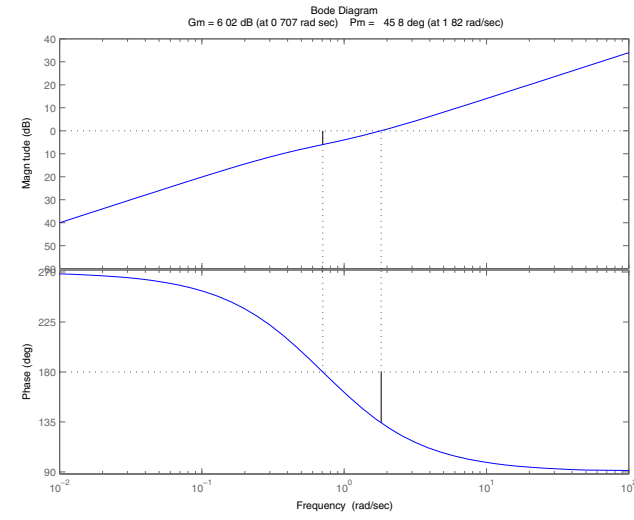

Fig. 6. Using bode plots to obtain the gain margin.

Definition 2: The stability margin against exponential averaging for the continuous-time closed-loop system, abbreviated as continuous exponential $A$-margin and denoted by $\lambda_{\max }(G)$, is the smallest $\lambda>0$ under which the closed-loop system becomes unstable. If the closed-loop system remains stable for all $\lambda>0$, we denote $\lambda_{\max }(G)=\infty$.

Suppose $G(s)=n(s) / d(s)$ where $n(s)$ and $d(s)$ are polynomial functions of $s$. Consider the characteristic equation of the closed-loop system

$$
1+F_{\lambda}(s) G(s)=1+\frac{1}{\lambda s+1} \frac{n(s)}{d(s)}=0
$$

or

$$
\lambda s d(s)+d(s)+n(s)=0
$$

which leads to

$$
1+\lambda \frac{s d(s)}{d(s)+n(s)}=0
$$

Theorem 2: The exponential A-margin $\lambda_{\max }(G)$ of $G(s)$ is the gain margin of

$$
H(s)=\frac{s d(s)}{d(s)+n(s)} .
$$

Example 1: Suppose $G(s)=(s+2) /(s-1)$. Then,

$$
H(s)=\frac{s d(s)}{d(s)+n(s)}=\frac{s^{2}-s}{2 s+1} .
$$

The gain margin can be obtained by using the Matlab function "margin" (which gives $\lambda_{\max }=2$ ) or by plotting the bode plot as shown in Figure 6 which gives $\lambda_{\max }=6.02 \mathrm{~dB}=2$.

\section{Performance Analysis}

The continuous time close-loop system equation is:

$$
y=\frac{G}{1+F_{\lambda} G} r+\frac{F_{\lambda} G}{1+F_{\lambda} G} d
$$

Here, we denote

$$
H_{\lambda}=\frac{-F_{\lambda} G}{1+F_{\lambda} G}
$$

If $d$ is a white noise, noise attenuation aims to reduce the $H^{2}$ norm of

$$
\eta=\inf _{0<\lambda<\lambda_{\max }}\left\|H_{\lambda}\right\|_{2}
$$


By minizing the $H^{2}$ norm of $H_{\lambda}$ respecting to $\lambda$, the optimal window size within the A-margin can be obtained. From the relationship $\alpha=e^{-T / \lambda}$, when $\lambda$ is optimized, increasing sampling rate will result in a larger optimal $\alpha$ for discrete time system.

\section{E. Fast Sampling for Disturbance Attenuation}

The continuous-time system $H_{\lambda}$ with impulse response $h_{\lambda}(t)$ is stable. For small $T$,

$$
\left\|H_{\lambda}\right\|_{2}^{2}=\int_{0}^{\infty} h_{\lambda}^{2}(t) d t \approx T \sum_{k=0}^{\infty} h_{\lambda}^{2}(k T) .
$$

Denoting sampled system and its pulse response as $\widetilde{H}_{\lambda}$ and $\widetilde{h}_{\lambda}$, we have $\widetilde{h}_{\lambda k}=T h_{\lambda}(k T)$ and

$$
\left\|\widetilde{H}_{\lambda}\right\|_{2}^{2}=\left\|\widetilde{g}_{\lambda k}\right\|_{2}^{2}=T^{2} \sum_{k=0}^{\infty} h_{\lambda}^{2}(k T)=T\left\|H_{\lambda}\right\|_{2}^{2}
$$

From

$$
y_{k}=\sum_{i=0}^{k} \widetilde{h}_{\lambda(k-i)} d_{i}
$$

if $d_{k}$ is i.i.d., zero mean and variance $\sigma^{2}$, then

$$
\begin{aligned}
\sigma_{k}^{2} & =E y_{k}^{2}=\sum_{i=0}^{k} \sum_{j=0}^{k} \widetilde{h}_{\lambda(k-i)} E d_{i} d_{j} \widetilde{h}_{\lambda(k-j)} \\
& =\sigma^{2} \sum_{i=0}^{k} \widetilde{h}_{\lambda(k-i)}^{2} \leq \sigma^{2}\left\|\widetilde{h}_{\lambda k}\right\|_{2}^{2}=\sigma^{2} T\left\|H_{\lambda}\right\|_{2}^{2}
\end{aligned}
$$

In fact,

$$
\sigma_{\max }^{2}=\sup _{k} \sigma_{k}^{2} \approx \sigma^{2} T\left\|H_{\lambda}\right\|_{2}^{2} .
$$

If $\left\|H_{\lambda}\right\|_{2}^{2}$ is optimized, then $\left\|H_{\lambda}\right\|_{2}^{2}=\eta$ as in (16). Consequently, the noise reduction ratio can be expressed as

$$
\widetilde{\eta}=T \eta
$$

\section{Control with Signal Averaging}

We now apply above findings to anesthesia control systems. The open-loop transfer function in (2) can be derived as

$$
G(z)=\frac{N(z)}{D(z)}
$$

with

$$
\begin{aligned}
N(z)= & 0.02311 z^{7}-0.09699 z^{6}-0.01243 z^{5}+0.4466 z^{4} \\
& -0.689 z^{3}+0.5101 z^{2}-0.2005 z+0.02235
\end{aligned}
$$

and

$$
\begin{aligned}
D(z)= & z^{12}-4.5 z^{11}+9.248 z^{10}-11.48 z^{9}+9.576 z^{8} \\
& -5.684 z^{7}+2.528 z^{6}-0.7518 z^{5}-0.2721 z^{4} \\
& +0.6608 z^{3}-0.507 z^{2}+0.2003 z-0.02234
\end{aligned}
$$

The open loop system is unstable.

When an exponential weighted filter is inserted for signal averaging, the closed-loop system's stability concerns have already been depicted in Figure 4. The closed-loop system's $H^{2}$

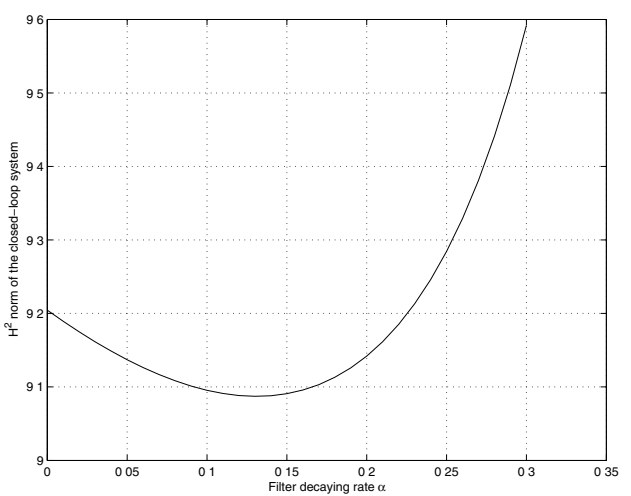

Fig. 7. Closed-loop system performance vs. filter decaying rates

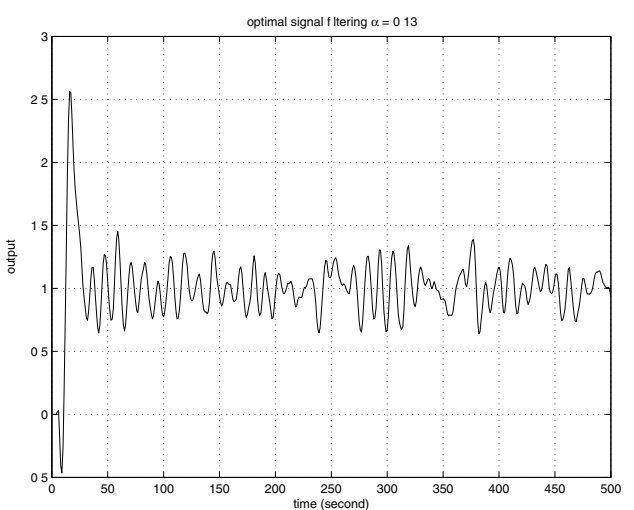

Fig. 8. Step response of the closed-loop system when the filter is optimally selected, and sampling interval $T=1$

norm, which defines the system's ability in noise attenuation, is shown in Figure 7.

The optimal filter decaying rate is $\alpha_{o p t}=0.1300$ with the corresponding $H^{2}$ norm 9.0872. The closed-loop system's step response is simulated when the filter is optimally selected and shown in Figure 8.

To relate this to re-sampling, we note that the above model is derived with the sampling interval $T=1$ second. From the relationship, $\alpha_{o p t}=e^{-T / \lambda_{o p t}}=e^{-1 / \lambda_{o p t}}$, we obtain $\lambda_{o p t}=$ 0.49 . This leads to the optimal choice of decaying rate when the sampling interval $T$ is reduced from 1 as

$$
\alpha=e^{-T / \lambda_{o p t}}=e^{-T / 0.49}=e^{-2.04 T} .
$$

When re-sampling is performed with $T \ll 1$, the $H^{2}$ norm of the closed-loop system will be reduced to $9.0872 \mathrm{~T}$ as established in (18). For reduced sampling intervals, improvements of noise attenuation are illustrated in Figure 9.

\section{CONCLUSION}

The impact of communication channels on feedback control in anesthesia applications in wireless based systems was investigated in this paper. The exponential window with decaying weighting of rate $\alpha, 0<\alpha<1$ is used to carry out our analysis. Our results show that the length of averaging window 

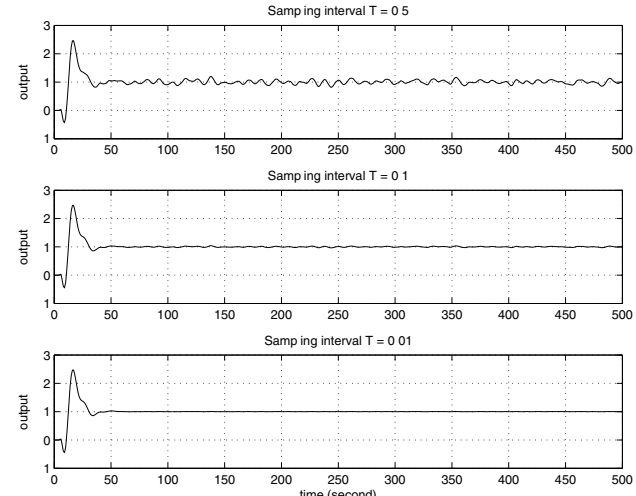

Fig. 9. The closed-loop system performance for reduced sampling intervals

has obvious negative impact on the performance of the closeloop systems. When the averaging window size is larger than some value, which is defined as the stability margin, closeloop systems will become unstable. In this paper, we derived some optimal strategies for choosing the window size of the close-loop systems. Our analysis shows that although the noise deduction performance of continuous time close-loop system with optimal averaging filter can not be improved, it can be achieved for a sampled system by increasing the sampling rate. Some simulations results of the feedback control with signal averaging are given to demonstrate our conclusions.

Our analysis is conducted on the basis of linear systems. Actually, anesthesia patient models show much nonlinearity. Our future works will consider the analysis of nonlinear systems.

\section{REFERENCES}

[1] S. L. Talbot,B. F. Boroujeny, Spectral Method of Blind Carrier Tracking for OFDM, IEEE Transactions on Signal Processing, VOL. 56, NO. 7, pp. 2706-2717, Jul. 2008

[2] E. Bataillou, E. Thierry, H. Rix, and 0. Meste, Weighted averaging using adaptive estimation of the weights, Signal Processing, VOL.44, Issue 1, pp. 51-66, Jun. 1995.

[3] C. Dong, J. Kehoe, J. Henry, E.C. Ifeachor, C.D. Reeve, and J.R. Sneyd, Closed-loop Computer Controlled Sedation with Propofol, Proc. of the Anaesthetic Research Society, 631P, 1999.

[4] J.C. Eisenach, Reports of Scientific Meetings - Workshop on Safe Feedback Control of Anesthetic Drug Delivery, Anesthesilogy, VOL. 91, pp. 600-601, August 1999.

[5] T.J. Gan, P.S. Glass, A. Windsor, F. Payne, C. Rosow, P. Sebel, and P. Manberg, Bispectral Index Monitoring Allows Faster Emergence and Improved Recovery from Propofol, Alfentanil, and Nitrous Oxide Anesthesia, Anesthesiology, VOL. 87, pp. 808-815, 1997.

[6] D.A. Linkens, Adaptive and Intelligent Control in Anesthesia, IEEE Control Systems Magazine, VOL. 12, Issue 6, pp. 6-11, Dec. 1992.

[7] L. Ljung and T. Söderström, Theory and Practice of Recursive Identification, MIT Press, Cambridge, MA, 1983.

[8] M. Milanese and A. Vicino,Optimal estimation theory for dynamic systems with set membership uncertainty: An overview, Automatica, VOL. 27, pp 997-1009, 1991.

[9] C. Rosow and P.J. Manberg,Bispectral Index Monitoring, Annual of Anesthetic Pharmacology, VOL. 2, pp. 1084-2098, 1998.

[10] L.Y. Wang and H. Wang, Control-oriented modeling of BIS-based patient response to anesthesia infusion, 2002 Internat. Conf. Math. Eng. Techniques in Medicine and Bio. Sci., Las Vegas, Jun. 24-27, 2002.
[11] L.Y. Wang and H. Wang,Feedback and predictive control of anesthesia infusion using control-oriented patient models, 2002 Internat. Conf. Math. Eng. Techniques in Medicine and Bio. Sci., Las Vegas, Jun. 24-27, 2002.

[12] L.Y. Wang, H. Wang, and G. Yin,Anesthesia infusion models: Knowledge-based real-time identification via stochastic approximation, 41st IEEE Cont. and Dec. Conf., Las Vegas, 2002.

[13] L.Y. Wang, H. Wang, and G. Yin, Reliable nonlinear identification in medical applications, 13th IFAC Symposium on System Identification (SysID 03), Rotterdam, The Netherlands, Aug. 27-29, 2003.

[14] X.-S. Zhang, R.J. Roy, and J.W. Huang, Closed-loop System for Total Intravenous Anesthesia by Simultaneously Administering Two Anesthetic Drugs, Proc. of the 20th Annual International Conference of the IEEE Engineering in Medicine and Biology, pp. 3052-3055, 1998. 